\title{
Evaluation of the Feasibility of a Web-Based Survey to Assess Patient-Reported Symptom Improvement and Treatment Satisfaction Among Patients with Psoriatic Arthritis Receiving Secukinumab
}

\author{
Marina Magrey ${ }^{1} \cdot$ Michael Bozyczko $^{2} \cdot$ Daniel Wolin $^{3} \cdot$ Margaret Mordin $^{3} \cdot$ Lori McLeod $^{3} \cdot$ Eric Davenport $^{3}$. \\ Costel Chirila ${ }^{3} \cdot$ Peter Hur ${ }^{4}$
}

Published online: 23 September 2019

(c) The Author(s) 2019

\begin{abstract}
Background and Objective Patient perspectives regarding treatment experience and satisfaction may be useful for clinicians when making treatment strategies. This US-based study assessed the feasibility of evaluating real-world, patient-reported narratives regarding symptom improvement and treatment satisfaction among patients with psoriatic arthritis treated with secukinumab.

Methods A cross-sectional, web-based survey collected data on demographics, disease characteristics, symptoms before and after secukinumab use, and treatment satisfaction with secukinumab.

Results Of 2755 patients screened, 200 patients with psoriatic arthritis were eligible and included in the analysis. Their mean age was 36.0 (standard deviation, 10.0 ) years; $55.5 \%$ were male and $75.0 \%$ were white. Most (87.5\%) were biologic experienced; the primary reason for discontinuation of their previous treatment was lack of effectiveness (28.6\%). Most patients (79.9\%) reported overall psoriatic arthritis symptom improvement after secukinumab initiation compared with before secukinumab initiation; a similar trend was observed for all individual symptoms evaluated. Approximately half of patients reported improvement within 4 weeks after starting secukinumab treatment, and $>90 \%$ reported improvement within 6 months. Most patients ( $\geq 96 \%$ ) expressed overall satisfaction with secukinumab regarding symptom improvement, speed of symptom improvement, frequency of administration, method of administration, ease of use, patient support services, and side effects, if any.

Conclusions Patient-reported perspectives may be feasibly collected to provide insights into treatment experience and satisfaction of secukinumab. Most patients with psoriatic arthritis in our real-world study experienced symptom improvement after initiating secukinumab; $>50 \%$ of patients reported symptom improvement within 4 weeks. Additionally, almost all patients reported satisfaction with secukinumab treatment.
\end{abstract}

\section{Key Points}

Electronic supplementary material The online version of this article (https://doi.org/10.1007/s40261-019-00856-8) contains supplementary material, which is available to authorized users.

Marina Magrey

mmagrey@metrohealth.org

1 Division of Rheumatology, The MetroHealth System and School of Medicine, Case Western Reserve University, 2500 MetroHealth Dr, Cleveland, OH 44109, USA

2 National Psoriasis Foundation, Portland, OR, USA

3 RTI Health Solutions, Research Triangle Park, NC, USA

4 Novartis Pharmaceuticals Corporation, East Hanover, NJ, USA
We explored the feasibility of assessing real-world experiences regarding overall treatment and satisfaction with secukinumab among patients with psoriatic arthritis

The majority of patients reported psoriatic arthritis symptom improvement within 6 months of secukinumab initiation and most patients expressed satisfaction with secukinumab treatment

Our data indicate that assessing patient-reported perspectives of patients with psoriatic arthritis may be a practical means to provide insights into treatment experience and satisfaction 


\section{Introduction}

Psoriatic arthritis (PsA) is a chronic inflammatory disease of the skin and musculoskeletal system [1], with an estimated prevalence of $0.25 \%$ in the USA [2]. Clinically, the disease is heterogenous, with both articular and extra-articular manifestations, which can complicate treatment. Psoriatic arthritis is frequently associated with psoriasis; up to $30 \%$ of patients with psoriasis - approximately $0.3-1.0 \%$ of the global population - may have a concurrent diagnosis of PsA [1, 3-5]. The Group for Research and Assessment of Psoriasis and Psoriatic Arthritis recognizes six clinical domains of PsA: peripheral disease, axial disease, enthesitis, dactylitis, skin disease, and nail disease; treatment guidelines recommend strategies that improve disease activity in all domains [6]. A better understanding of the roles of cytokines such as interleukin (IL)-17 and IL-23 in the pathogenesis of PsA and the research of treatments with new mechanisms of action such as IL-17 and IL-23 antagonists have led to the availability of newer therapeutic options that have improved patient outcomes.

Secukinumab, a fully human IgG1 monoclonal antibody that selectively binds to and neutralizes IL-17A, is a biologic approved in the USA for the treatment of psoriasis, PsA, and ankylosing spondylitis (AS) [7]. Secukinumab has demonstrated efficacy in the clinical domains of PsA [8-10]; the treatment of patients with PsA with secukinumab in phase III studies led to significantly greater improvements in American College of Rheumatology response criteria, skin and nail psoriasis, enthesitis, dactylitis, physical functioning, and quality of life, and a greater inhibition of radiographic structural progression [7, 11-13]. Response rates to secukinumab were sustained for up to 5 years of treatment in PsA [14], demonstrating a consistent long-term efficacy and safety profile. Taken together, secukinumab improves multiple domains of PsA and inhibits the progression of joint structural damage, thus enabling patients to better perform their daily activities.

There is limited evidence regarding the impact of secukinumab treatment in patients with PsA in real-world settings. Additionally, treatment satisfaction is not usually assessed in randomized controlled trials (RCTs). The consideration of patient perspectives on treatment outcomes is important to assess the effectiveness of the treatment in the real world [15]. In this exploratory study, we sought to determine the feasibility of collecting and evaluating real-world patient accounts of PsA treatment experience.

\section{Methods}

\subsection{Study Design and Population}

Patient data were compiled from a cross-sectional web-based survey in the USA. Patients with PsA or AS from patient panels hosted by Survey Sampling International were invited by e-mail, at random, to take part in the online survey. Eligibility criteria included the ability to read and sign informed consent, age $\geq 18$ years with a self-reported diagnosis of active PsA, initiation of secukinumab $\geq 3$ months before survey participation and receipt of the drug continuously since initiation, and willingness to complete the online survey; self-reported eligibility was first confirmed upon completion of a screener module prior to the full survey. A separate survey for AS was administered to patients meeting the eligibility criteria for AS; results from the AS analysis are reported elsewhere [16]. The invitation for survey participation included a unique survey link, embedded password for access to the patient survey, and a unique random user identification number to ensure that each eligible participant completed the questionnaire only once. No personal identifiers were stored with the survey responses. Informed consent was obtained electronically from eligible patients interested in participating in the PsA survey. Thus, the study was exempt from institutional review board approval.

The survey questionnaire was developed under the guidance of a rheumatologist in clinical practice (first author) and a patient with PsA (second author). Their real-world experience provided insight into PsA symptoms and the associated impact of those symptoms that resonate most with patients. Their input facilitated the development of a questionnaire that allowed patients to describe their experience in a meaningful manner. Data collected included demographics, clinical characteristics, medication history (taken over the past 12 months and immediately before initiation of secukinumab), PsA symptoms (including joint pain or tenderness, pain disrupting sleep, swelling of entire fingers or toes [indicating dactylitis], fatigue, morning stiffness, soreness in areas other than the joints, and ankle and heel pain [indicating enthesitis]), and patient treatment satisfaction with secukinumab. Patient-reported disease activity and functional status at the time of survey participation were measured by the Multi-Dimensional Health Assessment Questionnaire. The Routine Assessment of Patient Index Data 3 (RAPID3), which measures function, pain, and patient global estimate of status, is part of the MultiDimensional Health Assessment Questionnaire metric and is reported in this study. RAPID3 is scored from 0 to 10 ; a higher score indicates greater severity of disease [17]. A copy of the questionnaire is provided as Electronic Supplementary Material. 
Patients were asked about the aforementioned symptoms before and after secukinumab treatment for PsA and the time to symptom improvement. They were also assessed on their satisfaction with secukinumab treatment with regard to symptom improvement, speed of symptom improvement, frequency of treatment, method of administration, ease of use, side effects, and patient support services. Patients denoted if they were "very satisfied," "mostly satisfied," "somewhat satisfied," or "not satisfied" with these factors; patients were considered satisfied if they indicated "very," "mostly," or "somewhat satisfied."

\subsection{Data Analysis}

Patient demographics, self-reported disease severity, treatment history, PsA symptoms, and treatment satisfaction were descriptively analyzed for all patients included in the study. Mean, standard deviation (SD), median, interquartile range, and range were analyzed for continuous- or ordinaltype data. Frequencies and percentages were reported for categorical data. Each question was analyzed individually without imputation of item-level missing data; no statistical hypothesis testing or sample weighting of questionnaire responses was performed in this study.

\section{Results}

\subsection{Patient Demographics and Clinical Characteristics}

Of 2755 patients screened for both the PsA and AS surveys, 269 patients with PsA were eligible for the PsA survey. Of the 269 eligible patients, 266 completed the survey and three did not consent to participate; 66 patients had concurrent AS and were included in the AS survey analysis and excluded from the PsA survey analysis. A total of 200 patients with PsA who initiated secukinumab were included in the analysis. Patient demographics and clinical characteristics are shown in Table 1. The mean (SD) age of included participants was 36.0 (10.0) years; $55.5 \%$ were male, and $75.0 \%$ were white. Patients reported a mean (SD) time since symptom onset of 5.2 (5.4) years, with a mean (SD) time since diagnosis of $4.0(4.5)$ years. The mean age (SD) of these patients at the time of PsA diagnosis was 31.6 (10.2) years. All patients reported one or more comorbidities, most commonly anxiety (38.0\%), depression (29.0\%), and plaque psoriasis (28.0\%); $74.5 \%$ of patients reported deformities due to PsA. Patients had a mean (SD) RAPID3 score of 4.3 (1.8); 113 of 200 patients (56.5\%) reported RAPID3 scores of $>4$.

\subsection{Treatment and Medication History}

The treatment and medication history of patients in this study are summarized in Table 2. During the 12 months prior to secukinumab initiation, the majority of patients received one or more treatments for the disease, with $94.0 \%$ of patients receiving a non-biologic therapy and $87.5 \%$ of patients receiving a biologic therapy;

Table 1 Summary of patient demographics and disease characteristics among patients with psoriatic arthritis (PsA) who have been receiving secukinumab for $\geq 3$ months

\begin{tabular}{|c|c|}
\hline Characteristics & $\begin{array}{l}\text { Patients with } \\
\text { PsA }(N=200)\end{array}$ \\
\hline Age, mean (SD), years & $36.0(10.0)$ \\
\hline Female, $n(\%)$ & $89(44.5)$ \\
\hline \multicolumn{2}{|l|}{ Race and/or ethnicity, $n(\%)$} \\
\hline White & $150(75.0)$ \\
\hline Hispanic & $40(20.0)$ \\
\hline Black/African American & $27(13.5)$ \\
\hline Other & $15(7.5)$ \\
\hline \multicolumn{2}{|l|}{ Region, $n(\%)$} \\
\hline East & $44(22.0)$ \\
\hline South & $54(27.0)$ \\
\hline Midwest & $47(23.5)$ \\
\hline West & $44(22.0)$ \\
\hline North & $11(5.5)$ \\
\hline \multicolumn{2}{|l|}{ Employment status, $n(\%)$} \\
\hline Full time & $153(76.5)$ \\
\hline Part time & $19(9.5)$ \\
\hline Work at home & $5(2.5)$ \\
\hline Student & $7(3.5)$ \\
\hline Retired & $4(2.0)$ \\
\hline Disabled/unable to work & $12(6.0)$ \\
\hline Time since symptom onset, mean (SD), years & $5.2(5.4)$ \\
\hline Time since diagnosis, mean (SD), years & $4.0(4.5)$ \\
\hline \multicolumn{2}{|l|}{ Highest level of education } \\
\hline High school equivalent & $21(10.5)$ \\
\hline Some college, but no degree & $42(21.0)$ \\
\hline $\begin{array}{l}\text { College degree, advanced degree, or professional } \\
\text { degree }\end{array}$ & $137(68.5)$ \\
\hline \multicolumn{2}{|l|}{ Current health conditions, $n(\%)^{\mathrm{a}}$} \\
\hline Anxiety & $76(38.0)$ \\
\hline Chronic pain & $52(26.0)$ \\
\hline Depression & $58(29.0)$ \\
\hline Diabetes mellitus & $44(22.0)$ \\
\hline Fatigue & $55(27.5)$ \\
\hline Plaque psoriasis & $56(28.0)$ \\
\hline RAPID3 score, mean (SD) & $4.3(1.8)$ \\
\hline
\end{tabular}

RAPID3 Routine Assessment of Patient Index Data 3, SD standard deviation

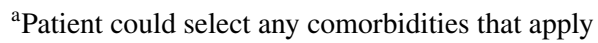


only one patient was treatment naive prior to initiating secukinumab. Among treatment-experienced patients, $75(37.5 \%)$ waited $<1$ month before initiating secukinumab, 90 (45.0\%) waited 1-4 months, and 31 (15.8\%) waited $\geq 5$ months. Among patients who had received prior treatment, $28.6 \%$ reported that a lack of effectiveness was the main reason for discontinuing their last treatment; other reasons cited included side effects $(26.0 \%)$, painful injections/infusions (12.2\%), and fear of injections or needles $(8.7 \%)$ (Table 2$)$.

\subsection{Symptoms of Psoriatic Arthritis Before and After Secukinumab Initiation}

After initiation of treatment with secukinumab, a lower proportion of patients evaluated in this study reported experiencing PsA symptoms, i.e., joint pain or tenderness, pain disrupting sleep, swelling of entire fingers or

Table 2 Summary of treatment history among patients with psoriatic arthritis (PsA) who have been receiving secukinumab for $\geq 3$ months

\begin{tabular}{lc}
\hline & Patients with \\
& PsA $(N=200)$ \\
\hline Duration of secukinumab treatment for PsA, $n(\%)$ & \\
$3-6$ months & $89(44.5)$ \\
$7-12$ months & $66(33.0)$ \\
$>12$ months & $45(22.5)$ \\
Approximate time between stopping previous treatment and initiat- \\
ing secukinumab, $n$ (\%) \\
$<1$ week & $23(11.7)$ \\
1 week to $<3$ months & $124(62.0)$ \\
3 to $\geq 5$ months & $49(24.5)$ \\
Main reason for stopping previous treatment, $n(\%)^{\mathrm{a}}$ & \\
Lack/loss of efficacy/effectiveness & $56(28.6)$ \\
Unwanted side effects & $51(26.0)$ \\
Injection/infusion was painful & $24(12.2)$ \\
Anxiety or fear of injections/needles & $17(8.7)$ \\
Other reasons & \\
Past medication use, $n(\%)$ & $48(24.0)$ \\
Over-the-counter NSAIDs & \\
Prescription NSAIDs & $183(91.5)$ \\
Steroids & $153(76.5)$ \\
Apremilast & $121(60.5)$ \\
Biologics & $73(36.5)$ \\
Never taken any non-biologic treatment & $175(87.5)$ \\
\hline
\end{tabular}

NSAIDs non-steroidal anti-inflammatory drugs

${ }^{a}$ Among patients who received a previous treatment for PsA $(n=196)$

bother reasons include "insurance would not pay;" "treatment was not easy or convenient to take;" "co-pay was too high;" "frequency of dosing was too high;" "number of required physician visits was too high;" "number of required blood tests was too high;" and "other" toes (indicating dactylitis), fatigue, morning stiffness, soreness in areas other than the joints, and ankle and heel pain (indicating enthesitis), compared with before secukinumab treatment (Fig. 1a). With regard to duration of secukinumab use, 89 patients $(44.5 \%)$ had been receiving secukinumab for 3-6 months and 111 patients (55.5\%) for $\geq 7$ months; symptom frequency with secukinumab treatment was lower in patients with a longer duration of use (Fig. 1b, c). Among patients experiencing PsA symptom improvements ("a little better," "moderately better," or "much better") since secukinumab initiation, $52.2 \%$ reported overall symptom improvement in PsA within 4 weeks after secukinumab initiation, $25.2 \%$ within 1-2 months, and $21.4 \%$ after 3 months; a similar trend was observed for all of the individual symptoms (Fig. 2).

Of 56 patients with psoriasis and PsA, 48 (85.7\%) reported better overall symptom control after secukinumab initiation compared with before secukinumab initiation ("a little better," 26.8\%; "moderately better," 39.3\%; "much better," $19.6 \%$ ); the majority of patients reported improvement in each PsA symptom assessed. Among the 48 patients reporting symptom improvement after secukinumab initiation, most $(62.5 \%)$ reported overall symptom improvement in PsA within 4 weeks after secukinumab initiation, $22.9 \%$ within $1-2$ months, and $10.4 \%$ after 3 months; a similar trend was observed for all of the individual symptoms.

\subsection{Treatment Satisfaction with Secukinumab}

As shown in Fig. 3, most patients with PsA reported overall satisfaction ("very satisfied," "mostly satisfied," or "somewhat satisfied") with secukinumab after $\geq 3$ months of treatment with respect to overall symptom improvement $(99.5 \%)$, speed of symptom improvement (100.0\%), frequency and method of administration (99.5\% and $97.0 \%$, respectively), ease of use (97.5\%), patient support services (98.0\%), and side effects, if any $(96.0 \%)$.

\section{Discussion}

In this study, we demonstrated the practicality of gathering real-world patient-reported experiences involving PsA symptom improvement and treatment satisfaction through a cross-sectional survey of patients with active PsA in the USA. The majority of the 200 secukinumabtreated patients with active PsA included in this study reported PsA symptom improvements and expressed treatment satisfaction with secukinumab in all PsA symptoms assessed in the survey. Approximately half of patients surveyed experienced overall improvement within 4 weeks 
A

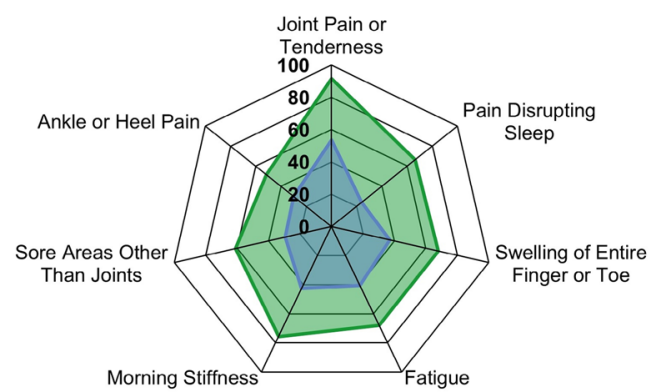

Overall Secukinumab Use $(\mathrm{N}=\mathbf{2 0 0})$
B

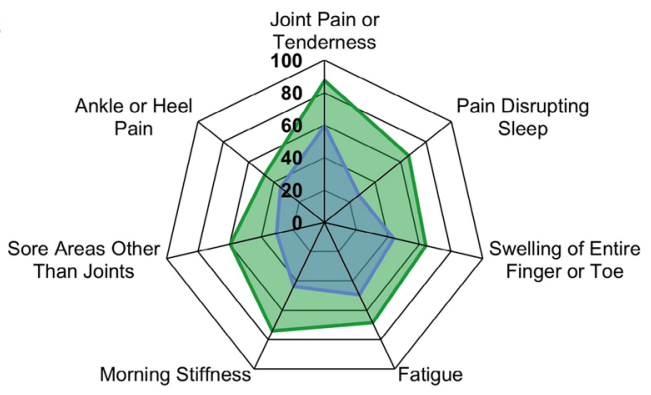

3 to 6 Months of Secukinumab Use $(n=89)$

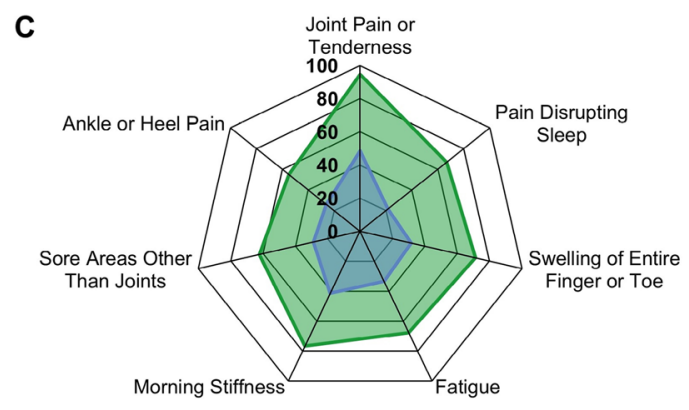

$\geq 7$ Months of Secukinumab Use $(n=111)$

Fig. 1 Proportion of patients experiencing psoriatic arthritis symptoms before and after initiating secukinumab in a the overall population $(N=200)$, b patients with 3-6 months of secukinumab use $(n=89)$, and $\mathbf{c}$ patients with $\geq 7$ months of secukinumab use $(n=111)$

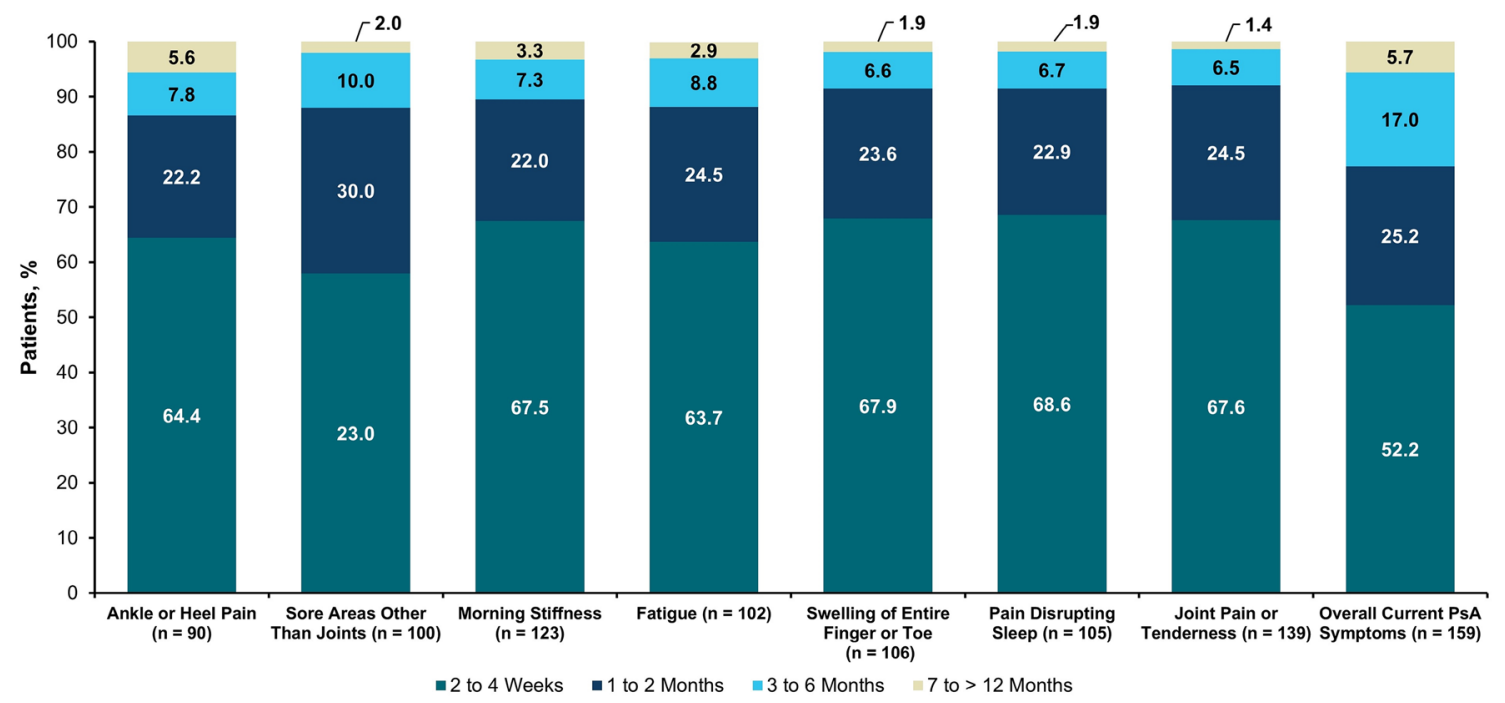

Fig. 2 Patient-reported time to first noticeable improvements in psoriatic arthritis (PsA) symptoms since secukinumab initiation $(N=200)$

of initiating secukinumab and $>94 \%$ within 6 months. Our survey questionnaire was developed with the guidance of a rheumatologist in clinical practice (first author) and a patient with PsA (second author). Their real-world familiarity with PsA symptoms and the associated impact of those symptoms contributed to the development of a practical questionnaire. Specifically, questionnaires and study-specific questions were selected based on their input to ensure that the wording was appropriate, and the content was relevant and meaningful to the patients.

Discrepancy between patient and physician perspectives regarding disease activity is common in rheumatic diseases [18] and is associated with increased disease burden and disability among patients with PsA [19]. Integrating clinician 


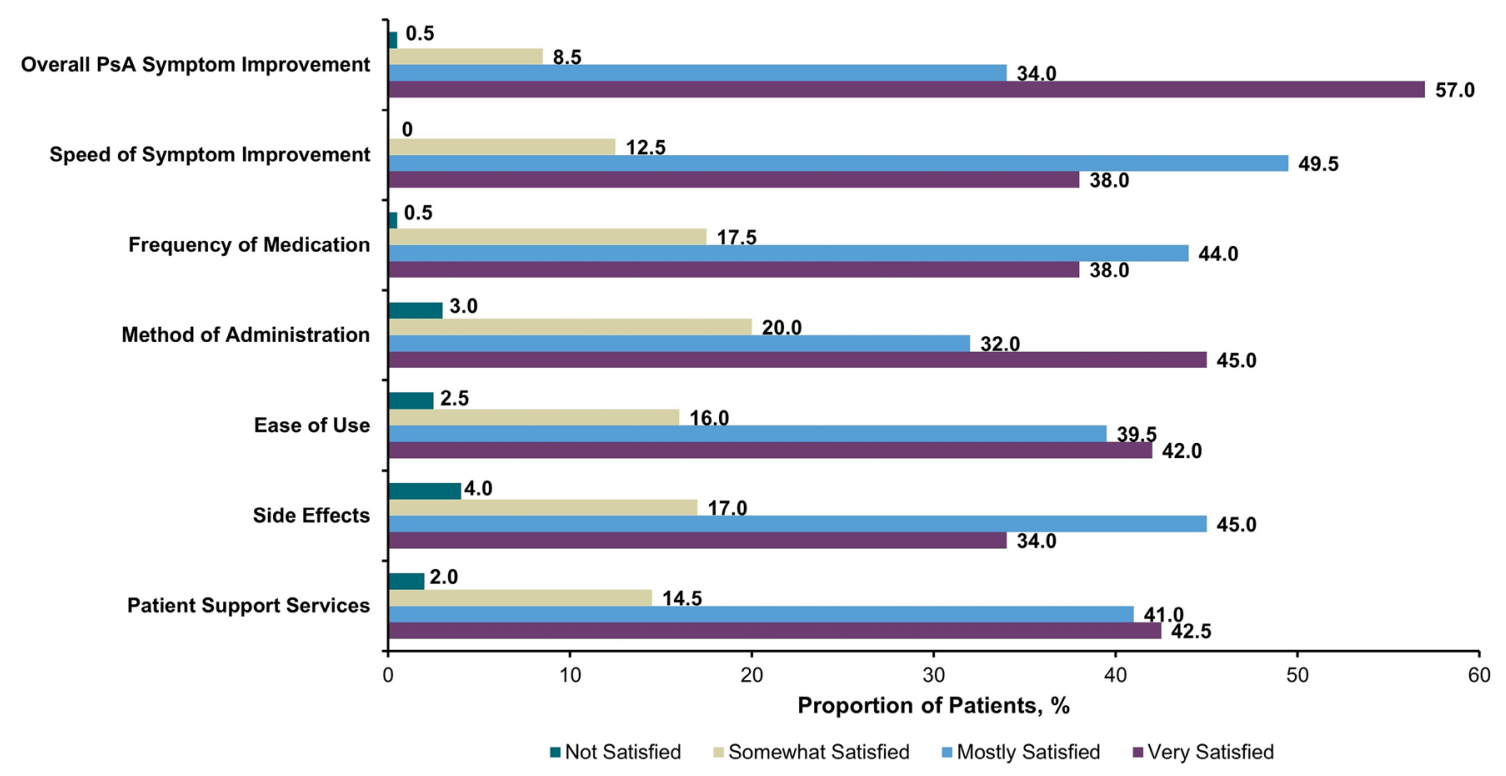

Fig. 3 Overall treatment satisfaction with secukinumab for psoriatic arthritis (PsA) $[N=200]$

perspectives and patient-reported measures (including patient perspectives on specific treatments) can result in more effective treatment strategies and improved patient outcomes [19]. Prior survey studies were conducted to evaluate patient experience with biologic therapies for rheumatic diseases. Although patient satisfaction was evaluated, these studies did not focus on specific biologics. National Psoriasis Foundation surveys administered from 2003 to 2011 showed that $52.3 \%$ of respondents with psoriasis and $45.5 \%$ with PsA were dissatisfied with their treatment, which comprised non-biologics and biologics, including etanercept, adalimumab, infliximab, ustekinumab, efalizumab, and infliximab [20]. Results from the Multinational Assessment of Psoriasis and Psoriatic Arthritis, a population-based survey of patients, dermatologists, and rheumatologists conducted between June and August 2012, showed that 25.2\% of surveyed patients with PsA reported dissatisfaction with the long-term safety of biologics [21]. The lack of satisfaction with therapy may negatively impact PsA management and treatment $[3,20,21]$. Optimizing satisfaction, therefore, is critical to improve the quality of healthcare [22, 23], adherence to treatment [20,24], and disease outcomes [20]. Our study is one of the first to evaluate patient-reported treatment experience and satisfaction with secukinumab. Most patients in our cohort expressed overall satisfaction with their secukinumab treatment experience. Understanding patient perspectives on specific treatments can help better inform clinicians when they are selecting biologic therapies for patients with PsA.

There are limited real-world studies on the effectiveness of secukinumab in patients with PsA. In two cohort studies that focused on physician-evaluated measures, treatment with secukinumab led to improvements in physician-evaluated pain, tender and swollen joint counts, and Disease Activity in Psoriatic Arthritis scores [25, 26]. Our study contributes to the pool of real-world knowledge on the effectiveness of secukinumab by identifying key PsA symptoms that were important to the patients and documenting improvements in these symptoms with secukinumab use, along with time to symptom improvement and overall patient satisfaction with secukinumab. Most patients in our study reported overall PsA symptom improvement as early as 4 weeks after secukinumab initiation compared with before secukinumab initiation, and a similar trend was observed for each symptom evaluated. The results of our study help address a knowledge gap by providing evidence of secukinumab effectiveness in a real-world patient population and insight into patient perspectives on secukinumab treatment. With regard to other IL-17A inhibitors, no real-world data on ixekizumab are available to date. Limited real-world data for the IL-12/23 inhibitor ustekinumab indicate that the biologic is effective and safe for the treatment of PsA, but patient perspectives were not assessed [27].

Further, patient-reported data collected from real-world studies can be more representative of the impact of treatment on patient daily lives compared with RCTs. While the results of our study pertaining to symptom improvement with secukinumab treatment are in agreement with those from phase III clinical studies [8-10], RCTs are conducted using select populations that may not be representative of patients seen in real-world clinical practice [28]. In our study, the majority of patients $(87.5 \%)$ were biologic experienced; in contrast, a high proportion of the patient populations in RCTs of secukinumab was biologic naive $[7,12,13]$. 
The patients in our study were also younger with a shorter time to diagnosis compared with patients typically enrolled in clinical trials [7, 12, 13, 29-31]. In addition, RCTs may not evaluate patient satisfaction with treatment. Our study provides perspective into how patients are evaluating their treatment in a real-world setting.

Our findings should be interpreted in the context of limitations inherent to patient surveys. Patient perspectives may be conditional on patient bias and experience. Additionally, participants may have been more likely to partake regularly in research studies and hence may have had greater interest in managing their disease than the general patient population. This study relied on patient self-report of PsA diagnosis, which may have led to under- or over-reporting of PsA symptoms. Physician-confirmed diagnosis and detailed treatment history were not available in this cross-sectional study. Patient experiences prior to secukinumab initiation were based on patient recall. Restricting the sample to patients who were being treated with secukinumab at the time of the survey may have created a selection bias. No inferences can be made on patient satisfaction and experience with secukinumab compared with specific previous treatments. Our current study did not include patients who had discontinued treatment with secukinumab prior to survey completion because the goal of this survey was to establish a set of questions that would be most meaningful to patients with regard to overall symptom improvement. In the future, we will examine patient symptom improvement and experience with secukinumab in comparison with other biologics, along with patient perspectives from secukinumab responders and non-responders.

\section{Conclusions}

Our study demonstrated the feasibility of collecting patient perspectives on treatment effectiveness and satisfaction using a questionnaire developed with clinician and patient input. Our results also indicate that the evaluation of PsA symptom improvement and treatment satisfaction as conducted in our study is directly applicable to a future longitudinal study to investigate these features among responders and non-responders to biologic therapies for PsA. The majority of patients with PsA in our study who were treated with secukinumab reported improvements in PsA symptoms with secukinumab and conveyed overall satisfaction with their secukinumab treatment experience. Additionally, half of patients surveyed experienced overall improvement within 4 weeks of initiating secukinumab and $>94 \%$ within 6 months. Management of PsA should encompass knowledge gained from clinical trials and real-world studies, including understanding the patient perspective. Assessment of symptom improvement and treatment satisfaction appears practical and applicable in our future studies for the evaluation of patient experience and satisfaction with PsA therapeutic agents.

Acknowledgements Support for third-party writing assistance for this article, furnished by Kheng Bekdache, PhD, of Health Interactions, Inc., was provided by Novartis Pharmaceuticals Corporation, East Hanover, NJ, USA.

\section{Compliance with Ethical Standards}

Funding This study was funded by Novartis Pharmaceuticals Corporation, East Hanover, NJ, USA.

Conflict of interest Marina Magrey has served as a consultant for Novartis. Michael Bozyczko has no conflicts of interest that are directly relevant to the content of this article. Daniel Wolin, Margaret Mordin, Lori McLeod, Eric Davenport, and Costel Chirila are employees of RTI Health Solutions. Peter Hur is an employee of Novartis.

Ethics approval An institutional review board exemption was obtained for the study prior to initiation of data collection.

Informed consent Informed consent was obtained electronically from eligible patients interested in participating in this PsA survey.

Data availability The data sets generated during and/or analyzed during the current study are proprietary and not publicly available.

Open Access This article is distributed under the terms of the Creative Commons Attribution-NonCommercial 4.0 International License (http://creativecommons.org/licenses/by-nc/4.0/), which permits any noncommercial use, distribution, and reproduction in any medium, provided you give appropriate credit to the original author(s) and the source, provide a link to the Creative Commons license, and indicate if changes were made.

\section{References}

1. Gladman DD, Antoni C, Mease P, et al. Psoriatic arthritis: epidemiology, clinical features, course, and outcome. Ann Rheum Dis. 2005;64:14-7.

2. Gelfand JM, Gladman DD, Mease PJ, et al. Epidemiology of psoriatic arthritis in the population of the United States. J Am Acad Dermatol. 2005;53:573.

3. Mease PJ, Gladman DD, Papp KA, et al. Prevalence of rheumatologist-diagnosed psoriatic arthritis in patients with psoriasis in European/North American dermatology clinics. J Am Acad Dermatol. 2013;69:729-35.

4. Gladman DD. Clinical features and diagnostic considerations in psoriatic arthritis. Rheum Dis Clin N Am. 2015;41:569-79.

5. Huynh D, Kavanaugh A. Psoriatic arthritis: current therapy and future approaches. Rheumatology (Oxford). 2015;54:20-8.

6. Coates LC, Kavanaugh A, Mease PJ, et al. Group for research and assessment of psoriasis and psoriatic arthritis 2015 treatment recommendations for psoriatic arthritis. Arthritis Rheumatol. 2016;68:1060-71.

7. Mease PJ, McInnes IB, Kirkham B, et al. Secukinumab inhibition of interleukin-17A in patients with psoriatic arthritis. N Engl J Med. 2015;373:1329-39. 
8. Orbai AM, de Wit M, Mease P, et al. International patient and physician consensus on a psoriatic arthritis core outcome set for clinical trials. Ann Rheum Dis. 2017;76:673-80.

9. Kirkham B, de Vlam K, Li W, et al. Early treatment of psoriatic arthritis is associated with improved patient-reported outcomes: findings from the etanercept PRESTA trial. Clin Exp Rheumatol. 2015;33:11-9.

10. Karreman MC, Weel AEAM, van der Ven M, et al. Performance of screening tools for psoriatic arthritis: a cross-sectional study in primary care. Rheumatology (Oxford). 2017;56:597-602.

11. McInnes IB, Mease PJ, Kirkham B, et al. Secukinumab, a human anti-interleukin-17A monoclonal antibody, in patients with psoriatic arthritis (FUTURE 2): a randomised, double-blind, placebocontrolled, phase 3 trial. Lancet. 2015;386:1137-46.

12. Strand V, Mease P, Gossec L, et al. Secukinumab improves patient-reported outcomes in subjects with active psoriatic arthritis: results from a randomised phase III trial (FUTURE 1). Ann Rheum Dis. 2017;76:203-7.

13. Mease $P$, van der Heijde $D$, Landewé $R$, et al. Secukinumab improves active psoriatic arthritis symptoms and inhibits radiographic progression: primary results from the randomised, double-blind, phase III FUTURE 5 study. Ann Rheum Dis. 2018;77:890-7.

14. Deodhar A, Mease PJ, McInnes IB, et al. Long-term safety of secukinumab in patients with moderate-to-severe plaque psoriasis, psoriatic arthritis, and ankylosing spondylitis: integrated pooled clinical trial and post-marketing surveillance data. Arthritis Res Ther. 2019;21:111.

15. Hsiao B, Fraenkel L. Incorporating the patient's perspective in outcomes research. Curr Opin Rheumatol. 2017;29:144-9.

16. Magrey M, Bozyczko M, Wolin D, et al. A pilot study to assess the feasibility of a web-based survey to examine patient-reported symptoms and satisfaction in patients with ankylosing spondylitis receiving secukinumab. Drugs Real World Outcomes. 2019;6:83-91.

17. Pincus T, Yazici Y, Bergman MJ. RAPID3, an index to assess and monitor patients with rheumatoid arthritis, without formal joint counts: similar results to DAS28 and CDAI in clinical trials and clinical care. Rheum Dis Clin North Am. 2009;35:773-8.

18. Lindstrom Egholm C, Krogh NS, Pincus T, et al. Discordance of global assessments by patient and physician is higher in female than in male patients regardless of the physician's sex: data on patients with rheumatoid arthritis, axial spondyloarthritis, and psoriatic arthritis from the DANBIO Registry. J Rheumatol. 2015;42:1781-5.
19. Furst DE, Tran M, Sullivan E, et al. Misalignment between physicians and patient satisfaction with psoriatic arthritis disease control. Clin Rheumatol. 2017;9:2045-54.

20. Armstrong AW, Robertson $\mathrm{AD}, \mathrm{Wu}$ J, et al. Undertreatment, treatment trends, and treatment dissatisfaction among patients with psoriasis and psoriatic arthritis in the United States: findings from the National Psoriasis Foundation surveys, 2003-2011. JAMA Dermatol. 2013;149:1180-5.

21. Lebwohl MG, Kavanaugh A, Armstrong AW, et al. US perspectives in the management of psoriasis and psoriatic arthritis: patient and physician results from the population-based Multinational Assessment of Psoriasis and Psoriatic Arthritis (MAPP) survey. Am J Clin Dermatol. 2016;17:87-97.

22. Cleary PD, McNeil BJ. Patient satisfaction as an indicator of quality care. Inquiry. 1988;25:25-36.

23. Rosenthal GE, Shannon SE. The use of patient perceptions in the evaluation of health-care delivery systems. Med Care. 1997;35:NS58-68.

24. Leung Y, Tam LS, Lee KW, et al. Involvement, satisfaction and unmet health care needs in patients with psoriatic arthritis. Rheumatology. 2009;48:53-6.

25. Robertson J, Tindell A, Crosbie D, Siebert S. 190 Real-world experience of secukinumab for psoriatic arthritis and axial spondyloarthritis. Rheumatology (Oxford). 2018. https://doi. org/10.1093/rheumatology/key075.414

26. Martinis F, Caimmi C, Carletto A, et al. Real-world efficacy and safety of secukinumab: data from Verona's cohort. Ann Rheum Dis. 2018;77:1544-5.

27. Queiro R, Coto-Segura P. Ustekinumab in psoriatic arthritis: need for studies from real-world evidence. Expert Opin Biol Ther. 2018;18:931-5.

28. Garrison LP Jr, Neumann PJ, Erickson P, et al. Using real-world data for coverage and payment decisions: the ISPOR Real-World Data Task Force report. Value Health. 2007;10:326-35.

29. Mease PJ, Gladman DD, Ritchlin CT, et al. Adalimumab for the treatment of patients with moderately to severely active psoriatic arthritis: results of a double-blind, randomized, placebo-controlled trial. Arthritis Rheum. 2005;52:3279-89.

30. Antoni C, Krueger GG, de Vlam K, et al. Infliximab improves signs and symptoms of psoriatic arthritis: results of the IMPACT 2 trial. Ann Rheum Dis. 2005;64:1150-7.

31. Kavanaugh A, van der Heijde D, McInnes IB, et al. Golimumab in psoriatic arthritis: one-year clinical efficacy, radiographic, and safety results from a phase III, randomized, placebo-controlled trial. Arthritis Rheum. 2012;64:2504-17. 